\title{
ISOLATION OF CELLULOSE NANOFIBRES FROM ARECA SPATHE AND ITS CHARACTERIZATION
}

\author{
Venugopal B \\ Department of Polymer Science and Rubber Technology, Cochin University of Science and \\ Technology, Kochi, 682022 Kerala, India \\ Department of Chemistry, Government College, Kattappana, 685508, Kerala, India \\ Jayalatha Gopalakrishnan* \\ Department of Chemistry, Government College, Kattappana, 685508, Kerala, India \\ *Corresponding Author's Email: gjayalatha@gmail.com
}

\begin{abstract}
Cellulose nanofibers have gained much interest because of its high mechanical properties, tunable surface groups, excellent strength/weight ratio, biodegradability and low cost. The present work deals with the isolation of cellulose nanofibres from Areca spathe which is an agricultural waste. Cellulose nanofibres can be conveniently extracted from Areca spathe by sulphuric acid hydrolysis. The steps involved chemomechanical methods such as grinding, homogenization, alkali treatment, bleaching and sulphuric acid hydrolysis. After alkali treatment and bleaching, the $\alpha$-cellulose content was found to increase tremendously. The removal of hemicellulose and lignin was confirmed by the FTIR studies. The increase in crystallinity was confirmed by the XRD analysis. Surface morphological studies using SEM revealed that the extracted fibres had a diameter of $55-70 \mathrm{~nm}$. Thermogravimetric analysis results showed that thermal stability improved significantly for bleached fibres compared to alkali-treated, nanofibres and raw fibres.
\end{abstract}

Key words: Arecaspathe, nanofibres, crystallinity, sulphuric acid hydrolysis.

Cite this Article: Venugopal B and Jayalatha Gopalakrishnan, Isolation of Cellulose Nanofibres from Areca Spathe and its Characterization, International Journal of Advanced Research in Engineering and Technology, 10 (3), 2019, pp 118-127.

http://iaeme.com/Home/issue/IJARET?Volume=10\&Issue $=3$

\section{INTRODUCTION}

Arecanut tree (Areca catechu) is one among the agro-industry plants in tropical and subtropical regions. Areca belongs to the species Areca catechu under Arecaceae family and believed to have originated in either Malaysia or Philippines. Areca plant is cultivated on a large scale in many places of India and Arecanut is used in the manufacture of paint, medicine, chewable gudka etc. Many parts of the plant can be used for extraction of cellulose and thereby 
nanocellulose because of the abundance of the plant in Kerala, India. Areca spathe has been made into disposable, biodegradable plates in some areas of Tamil Nadu, India. Most of the raw material goes as an agricultural waste and cause decay related issues in the plantation. This biological waste material if utilized can be a renewable feedstock for the production of value added products for special applications.

Cellulose nanofibre being a natural fibre could be considered as a potential substitute for synthetic fibres in almost all applications. Therefore the determination of the structural and morphological characteristics of the extracted cellulose nanofibre is essential. The lignocellulosic fiber has a complex structure and is mainly composed of cellulose, hemicellulose and lignin. The complex aromatic lignin and polysaccharides such as hemicelluloses and $\alpha$ - cellulose are generally resistant to common solvents. Among these biomaterials (cellulose, hemicellulose and lignin) cellulose has much importance because of its peculiar properties. Separation of pure cellulose from biomass components constitutes one of the major obstacles to efficient utilization of the renewable resource by different methods [1]. Cellulose thus obtained from the biomaterial can be converted into cellulose nanofibres by controlling the reagents and conditions used for nanomaterial extraction. In these circumstances Areca spathe can be an ideal raw material resource for cellulose nanofibre (AN) extraction.

In the present work, nanocellulose fibres were isolated from Arecaspathe using chemical and mechanical treatments. The fibres at different stages of treatment were analysed usingFTIR, XRD and TGA and SEM.

\section{EXPERIMENTAL}

\subsection{Materials}

Spathes of Areca palm were collected from an Areca farm in the western coastal region of Kerala, South India. The chemicals used to produce cellulose nanofibre include sodium hydroxide, sodium chlorite; acetic acid and sulphuric acid were supplied by Merck Specialties Private Ltd. Bengaluru. All the chemicals were of analytical grade.

\subsection{Isolation of cellulose nanofibres (AN)}

The procedure for the isolation of cellulose nanofibres is described in Figure 1.

\subsubsection{Alkali treatment}

The Areca spathe was washed, dried in sunlight and cut into small pieces having size less than $1 \mathrm{~cm}$ (AR). The fibres were treated with $5 \% \mathrm{NaOH}$ at $50{ }^{\circ} \mathrm{C}$ for $4 \mathrm{~h}$ with constant stirring. The treatment was repeated twice and then washed until free from alkali. The alkali treated fibres is designated as (AA).

\subsubsection{Bleaching process}

For removal of lignn, the fibres were ground into a pulp form using a kitchen grinder. The fibres were subjected to bleaching using a mixture of sodium chlorite $\left(\mathrm{NaClO}_{2}\right)$ and acetic acid for 2 $\mathrm{h}$ at $60^{\circ} \mathrm{C}$ [2]. This resulted in pure white fibres. The bleached fibres (AB) were further washed with de-ionised water until neutral $\mathrm{pH}$.

\subsubsection{Homogenization}

The bleached dispersion was homogenized using ULTRA - TURRAX disperser at $14000 \mathrm{rpm}$ for $30 \mathrm{~min}$. During this mechanical process microfibers were formed and get separated.

\subsubsection{Acid Hydrolysis}

The homogenized fibres was then subjected to sulphuric acid hydrolysis $(45 \% \mathrm{w} / \mathrm{w})$ at $45^{\circ} \mathrm{C}$ for $2 \mathrm{~h}$, followed by dilution with excess of water. The cellulose nanofibre (AN) dispersion thus obtained was centrifuged at 10,000 rpm for 5 min repeatedly, until it becomes neutral. 
Venugopal B and Jayalatha Gopalakrishnan

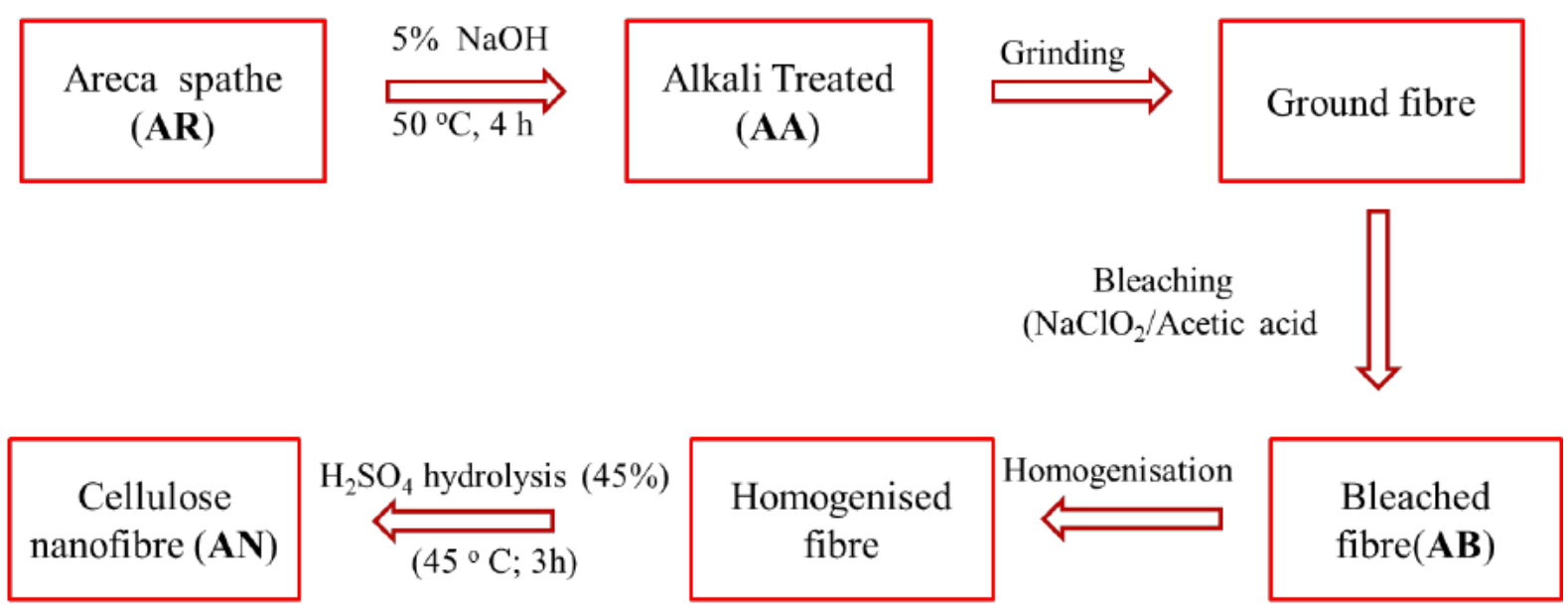

Figure 1. Cellulose nanofibre (AN) extraction flow chart

\section{CHARACTERIZATION}

\subsection{Chemical composition analysis}

The cellulose and lignin fractions of the fibres at different stages of processing were determined using Acid-detergent fibre (ADF) method [3]. Two replicated samples were analysed and the average values are reported.

\subsection{Fourier Transform Infrared spectroscopy (FTIR)}

FTIR spectra of the samples were recorded using Thermo Nicolet, Avatar 370 in the attenuated total reflection (ATR) mode. The changes in the functional group at different stages of treatment was analysed within the wavenumber range of $4000-400 \mathrm{~cm}^{-1}$ at a resolution of $4 \mathrm{~cm}^{-1}$.

\subsection{X-ray Diffraction analysis (XRD)}

$\mathrm{X}$-ray diffraction patterns of fibre samples at different stages of treatment were obtained by Bruker AXS D8 Advance X-ray powder diffractometer equipped with $\mathrm{Cu} \mathrm{K} \alpha$ radiation source operating at a voltage of $40 \mathrm{kV}$ and current of $35 \mathrm{~mA}$. The diffraction intensities were recorded from 3 to $80^{\circ}$ ( $2 \theta$ angle) at increments of $0.02^{\circ}$ at a wavelength of $1.541 \mathrm{~A}^{\circ}$. The crystallinity index $(\mathrm{CI})$ of the samples was calculated according to the following equation.

$$
\mathrm{CI}(\%)=\frac{\mathrm{I}_{200}-\mathrm{I}_{\mathrm{am}}}{\mathrm{I}_{200}} \times 100
$$

where $I_{200}$ is the peak intensity which arises from both amorphous and crystalline fractions and $\mathrm{I}_{\mathrm{am}}$ is the intensity of the amorphous fraction. The crystallite size (D) was calculated by the Debye Scherrer equation [4].

$$
\mathrm{D}=\frac{k \lambda}{\beta \cos \theta}
$$

where, $\mathrm{k}$ is a dimensionless shape factor and taken as $0.94, \lambda$ is the $\mathrm{X}$-ray wavelength $(0.154$ $\mathrm{nm}), \beta$ is the full width at half maximum (FWHM) of the peak, and $\theta$ is the Bragg's angle.

\subsection{Scanning Electron Microscopy}

SEM analysis of cellulose nanofibres was observed using a JEOL Model JSM 6390 LV scanning electron microscope at an accelerating voltage of $20 \mathrm{kV}$. To prevent charging, the sample was coated with gold using a vacuum sputter coater. 


\subsection{Thermogravimetric analysis (TGA)}

The extent of thermal decomposition of the cellulose fibres after each treatment was determined by using TA Instruments TGA Q50 under a nitrogen atmosphere with a flow rate of $40 \mathrm{ml} / \mathrm{min}$. The measurement was carried out from $30-800{ }^{\circ} \mathrm{C}$ at a heating rate of $20^{\circ} \mathrm{C} / \mathrm{min}$.

\section{RESULTS AND DISCUSSION}

\subsection{Chemical Composition}

Chemical constitution of the raw Areca spathe is given in Table 1. The cellulose content of Areca spathe fibre is about $39 \%$ which is comparable with that of many of the main plant cellulose sources reported in literature such as wheat straw (39\%), [5], rice straw (38\%) [6] coir fibres (43\%), Kenaf fibres (36\%), [7] Arecanut husk (34\%) [8], etc.

Table 1 Chemical constitution of Areca spathe

\begin{tabular}{|l|c|}
\hline Constituents & $\%(\mathrm{w} / \mathrm{w})$ \\
\hline$\alpha-$ Cellulose & $39.6 \pm 1.3$ \\
\hline Hemicelluloses & $25.2 \pm 2.1$ \\
\hline Lignin & $15.4 \pm 1.3$ \\
\hline Moisture & $8.8 \pm 1.2$ \\
\hline Pectin & $5.8 \pm 0.8$ \\
\hline Waxy/Fatty matter & $1.3 \pm 0.3$ \\
\hline Ash & $3.9 \pm 0.4$ \\
\hline
\end{tabular}

Table 2.Chemical composition of Areca spathe at different stages of treatment

\begin{tabular}{|l|c|c|c|}
\hline $\begin{array}{c}\text { Fibre at different } \\
\text { stages }\end{array}$ & $\begin{array}{c}\boldsymbol{\alpha} \text { - Cellulose } \\
(\mathbf{\%}, \mathbf{w} / \mathbf{w})\end{array}$ & $\begin{array}{c}\text { Hemicelluloses } \\
(\mathbf{\%}, \mathbf{w} / \mathbf{w})\end{array}$ & $\begin{array}{c}\text { Lignin } \\
\mathbf{( \% ,}, \mathbf{w} / \mathbf{w})\end{array}$ \\
\hline Raw (AR) & $39.6 \pm 1.3$ & $25.2 \pm 2.1$ & $15.4 \pm 1.3$ \\
\hline Alkali treated (AA) & $62.7 \pm 3.1$ & $6.2 \pm 0.5$ & $8.3 \pm 1.9$ \\
\hline Bleached (AB) & $84.5 \pm 5.4$ & $0.5 \pm 0.2$ & $3 \pm 0.9$ \\
\hline
\end{tabular}

Table 2 shows the percentage composition of $\alpha$-cellulose, hemicelluloses and lignin of Areca spathe fibres at different stages of treatment. From the table, it is clear that the raw fibre has the lowest percentage of cellulose content and highest percentage of hemicellulose and lignin content. When subjected to alkali treatment, the cellulose content significantly increases while the hemicellulose and lignin content decreases. Alkali, being a pre-swelling agent, increases the accessibility of the core material to further treatments. Alkali treatment starts the removal of hemicellulose and lignin by the breakdown of ether linkages between hemicellulose and lignin [9]. The hemicellulose content is reduced from $25.2 \%$ to $8.1 \%$ and the lignin content from $15.4 \%$ to $8.3 \%$ on two successive alkali treatments. In addition to hemicelluloses and lignin, pectin was also removed by alkali treatment [10]. The crystalline regions of the cellulose remain intact during chemical treatments. The grinding forces after alkali treatment helps the fibrillation by breaking the interlinking between cellulose fibres and other cementing materials. The fibrillation further facilitates the easy penetration of the bleaching agent and thereby removes lignin. Use of $\mathrm{NaClO}_{2}$ during bleaching reacts with lignin resulting in an oxidative fragmentation to form lignin chloride which is water soluble and is removed by filtration. After bleaching it is found that the percentage of cellulose content increased to $84.5 \%$ by the removal of non-cellulosic components.

On acid hydrolysis, the hydrolytic cleavage of glycosidic bond between the two anhydroglucose units occurs thus dissolving the amorphous regions and leaving behind the crystalline region [11]. The yield of cellulose nanofibre (AN) obtained from Areca spathe by sulphuric acid hydrolysis is $10-15 \%$. 


\subsection{FTIR Analysis}

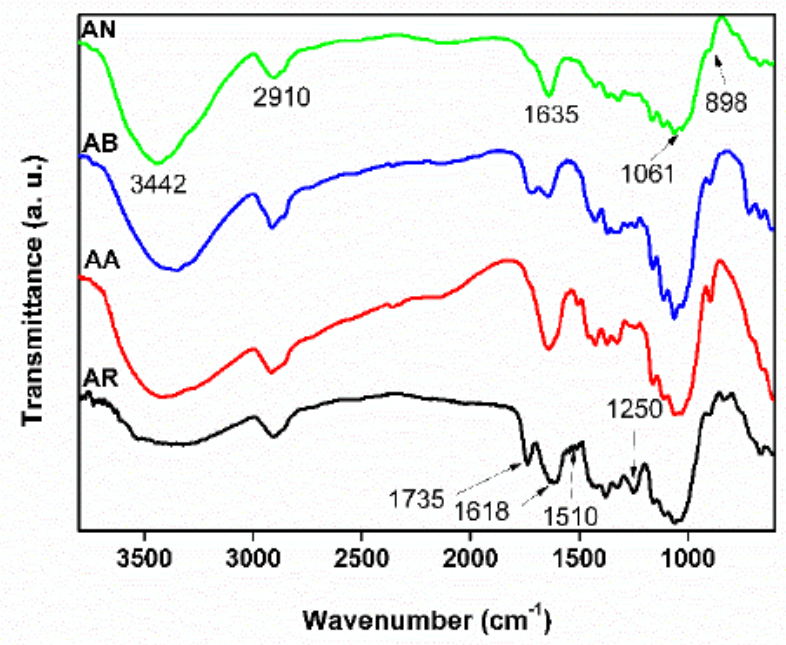

Figure 2. FTIR spectra of cellulose fibres at different stages of chemical treatment

The FTIR spectra of Areca spathe fibres have been taken for studying the changes in the chemical structures after various chemical treatments. FTIR spectra of the raw, alkali treated, bleached and acid hydrolysed fibres are shown in Figure 2. The absorption bands in the range $3340 \mathrm{~cm}^{-1}$ and $2910 \mathrm{~cm}^{-1}$ found in the spectra of all fibre samples represent the $\mathrm{O}-\mathrm{H}$ stretching vibrations of the hydrogen bonded hydroxyl group and the $\mathrm{C}-\mathrm{H}$ stretching of cellulose respectively $[12,13]$. The peak at $1735 \mathrm{~cm}^{-1}(-\mathrm{C}=\mathrm{O}$ stretching $)$ in raw fibre (AR) is due to the acetic and uronic ester groups of the hemicelluloses or the ester linkages of the carboxylic group of ferulic and p-coumaric acids of lignin or hemicellulose $[14,15]$. The peaks at $1618 \mathrm{~cm}^{-1}$ and $1510 \mathrm{~cm}^{-1}$ corresponding to the aromatic $\mathrm{C}=\mathrm{C}$ skeletal vibrations and $1250 \mathrm{~cm}^{-1}$ to the aromatic $\mathrm{C}-\mathrm{O}$ stretching of lignin found in the spectrum of raw fibre are not found in the bleached fibre (AB). Absence of these peaks in the spectrum of bleached fibre indicates the complete removal of hemicellulose and lignin. The peak at $1635 \mathrm{~cm}^{-1}$ in the treated fibres AA, AB and AN corresponds to the bending vibrations of adsorbed water. The peak at $1061 \mathrm{~cm}^{-1}$ and $898 \mathrm{~cm}^{-1}$ corresponds to the glucose ring stretching. The removal of lignin and hemicellulose indicates the increase in cellulosic content with different chemical treatments.

\subsection{Morphological Analysis}

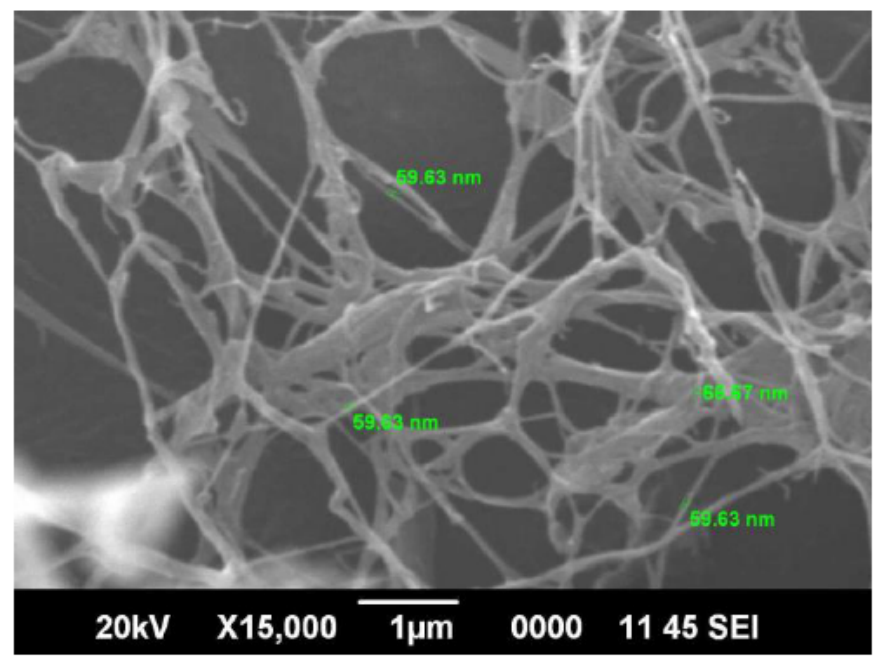

Figure 3.SEM images of cellulose nanofibre (AN) 
The morphology of the nanofibre obtained after chemo-mechanical treatments is shown in Figure 3. The image shows that the diameters of sulphuric acid hydrolysed fibres are in the range of $55-70 \mathrm{~nm}$ due to the removal of amorphous regions in the cellulosic chain. The cellulose nanofibrils are several microns in length. Some nanofibres shows larger diameter due to aggregation of nanofibres during drying the samples for SEM analysis.

\section{4. $X$ ray Diffraction studies}

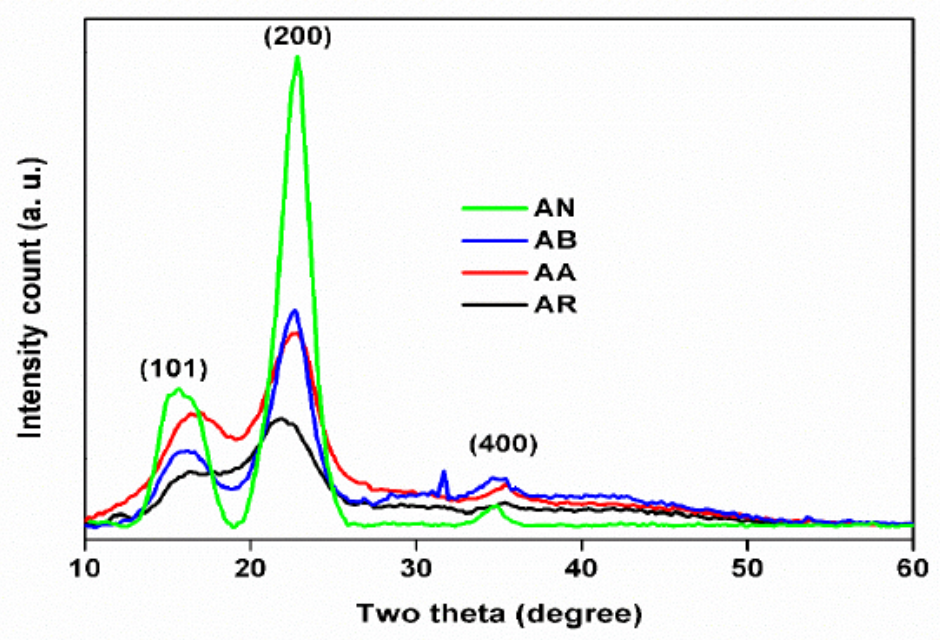

Figure 4. XRD patterns of raw (AR) and treated fibres (AA, AB, AN).

Figure 4 represents the XRD patterns of the raw fibre and the chemo-mechanically treated

Fibres. The crystalline structure of cellulose can be well studied by the XRD patterns. Characteristics peaks at two theta values $16^{\circ}, 22^{\circ}$ and $34^{\circ}$ corresponding to (101), (200) and (400) planes respectively found in the diffractograms of the samples at different stages of treatment is likely to be associated with the crystalline structure of cellulose I [16]. The ordered crystalline arrangements in the cellulose appears due to the formation of inter and intra molecular H-bonding by the hydroxyl groups [17]. The H-bonding restricts the free movement of the cellulosic chains and they align close together in an orderly manner which tends to induce crystallinity. The crystallinity indices of fibres at different stages are given in Table 3 . In the case of raw fibre (AR), the crystalline domains are embedded in the matrix of amorphous components like hemicelluloses, lignin and pectin thus a low crystallinity index of $44 \%$. The crystallinity increases with alkali treatment (AA) and bleaching (AB). This is attributed to the effective removal of the amorphous components.

Table 3 Crystallographic parameters of Areca spathefibre

\begin{tabular}{|c|c|c|}
\hline $\begin{array}{c}\text { Fibre at different } \\
\text { Stages }\end{array}$ & $\begin{array}{c}\text { Crystallite size } \\
(\mathbf{\AA})\end{array}$ & $\begin{array}{c}\text { Crystallinity } \\
\text { Index (\%) }\end{array}$ \\
\hline AR & 28.1 & 44 \\
\hline AA & 23.6 & 57 \\
\hline AB & 33.4 & 62 \\
\hline AN & 41.3 & 78 \\
\hline
\end{tabular}

The highest crystallinity index of nanofibres (AN) after acid hydrolysis is due to the fact that, randomly oriented amorphous regions in the cellulosic chain are easily prone to acid attack and the hydrolytic cleavage of glycosidic bonds produces individual crystallites [18]. The crystallite size and the crystallinity index with respect to (200) plane of each of the cellulosic 
samples were calculated from the X-ray diffraction peak profile and it is given in Table 3. It is clear that the crystallite size and crystallinity index get changed depending upon the chemo mechanical treatments.

\subsection{Thermogravimetric Analysis}

Thermogravimetric analysis was carried out to study the degradation characteristics of fibres at various stages of treatment. The TGA and DTG curves of raw fibre (AR), alkali treated fibre (AA), bleached fibres (AB) and sulphuric acid hydrolysed nanofibres (AN) are shown in Figure 5. Since different non- cellulosic and cellulosic components are present, the thermal degradation of raw fibre is a multi-stage process. Initial weight loss starting at $40{ }^{\circ} \mathrm{C}$ and continuing up to around $120^{\circ} \mathrm{C}$ for all the fibres is due to the evaporation of loosely bound moisture on the surface and the intermolecular hydrogen
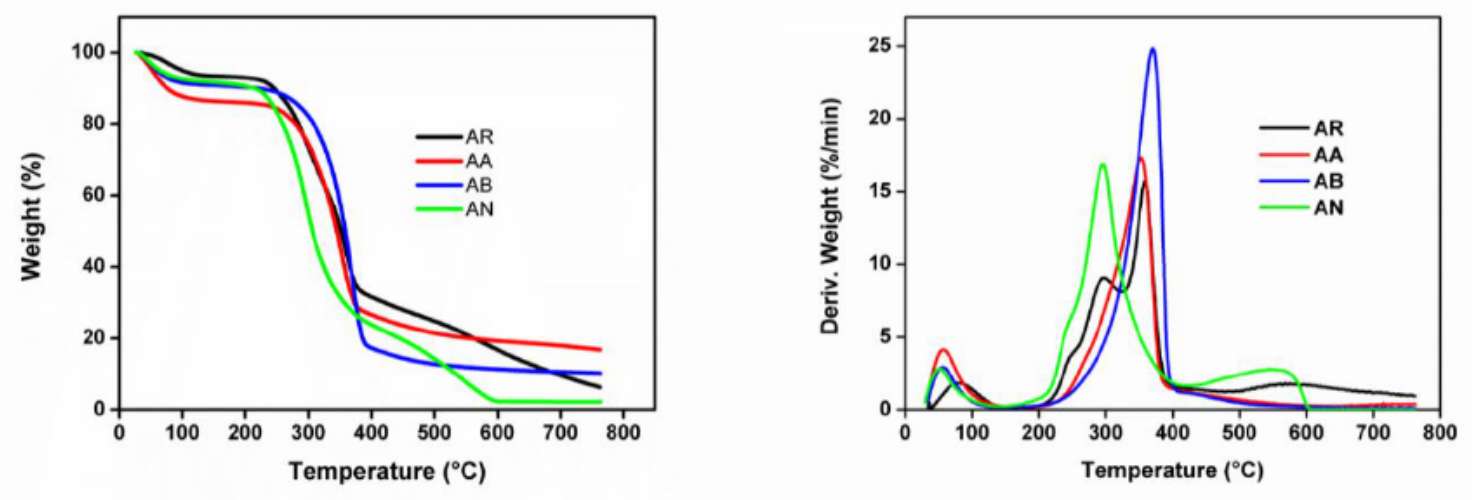

Figure 5. TGA and DTG curves of Areca spathe at different stages of chemical treatment

bonded chemisorbed water. The FTIR spectrum has confirmed the presence of the chemisorbed water. The onset decomposition temperature, maximum decomposition temperature and residue are given in Table 4. Since different non- cellulosic and cellulosic components are present, the thermal degradation of raw fibre (AR) is a multi-stage process. The DTG curve of AR shown in Figure 5b) shows a major decomposition peak at $358{ }^{\circ} \mathrm{C}$ and a shoulder peak at $280{ }^{\circ} \mathrm{C}$. The shoulder peak is caused by the thermal decomposition of hemicellulose and the main peak due to the breakdown of glycosidic linkages of cellulose. The third stage of degradation is likely to be associated with lignin which decomposes over a broader temperature range than cellulose and hemicelluloses. This broader decomposition range of lignin is because of the presence of various oxygen functional groups from its structure with different thermal stabilities and their cleavage occurring at different temperatures.

Table 4 Thermal degradation data of Areca spathe fibre at different stages of treatment

\begin{tabular}{|c|c|c|c|}
\hline $\begin{array}{c}\text { Sample } \\
\text { Code }\end{array}$ & $\begin{array}{c}\text { Onset } \\
\text { degradation } \\
\text { temperature }\left({ }^{\mathbf{0}} \mathbf{C}\right)\end{array}$ & $\begin{array}{c}\text { Max. Degradation } \\
\text { Temperature } \\
\left({ }^{\circ} \mathbf{C}\right)\end{array}$ & $\begin{array}{c}\text { Residue at } \\
\mathbf{6 0 0}^{\mathbf{}} \mathbf{C} \\
(\mathbf{w t .} \%)\end{array}$ \\
\hline AR & 210 & 358 & 16.8 \\
\hline AA & 221 & 358 & 19.3 \\
\hline AB & 225 & 370 & 11.3 \\
\hline AN & 205 & 296 & 3.1 \\
\hline
\end{tabular}

The degradation of alkali treated pulp starts at $221{ }^{\circ} \mathrm{C}$ and the rate of degradation reaches a maximum at $358^{\circ} \mathrm{C}$ owing to the decomposition of cellulose whereas the bleached fibre starts degradation at $225^{\circ} \mathrm{C}$ and a maximum degradation rate at $370^{\circ} \mathrm{C}$. The absence of shoulder peak in $\mathrm{AA}$ and $\mathrm{AB}$ confirms the effective removal of hemicellulose. The rise in the onset and 
degradation temperatures of bleached fibre is due to the removal of non - cellulosic components which makes the cellulose more dense and compact. The reduction in the thermal stability of AN may be the result of increase in the amount of short cellulose chains. Moreover, the negatively charged sulphonic acid groups present on the outer surface of cellulose fibres also resulted in the decrease of thermal stability [19] Sulphuric acid may hydrolyze the big cellulose chain into smaller ones [20]. The solid AN may possibly undergo direct solid-to-gas phase transitions catalyzed by surface sulphate groups around $320^{\circ} \mathrm{C}[21]$. The carbonaceous residues obtained at $600{ }^{\circ} \mathrm{C}$ for raw fibre is $16.8 \%$ and for alkali treated fibre, the residue increased to $19.3 \%$ because of the removal of thermally less stable hemicelluloses, whereas a relatively a low amount of residue $(11.3 \%)$ remains for the bleached and lowest for nanofibre $(3.1 \%)$. The highest residue in the raw fibre compared to that for the treated ones can be attributed to the presence of lignin, which have very low degradation rates.

\section{CONCLUSIONS}

The cellulose nanofibres were successfully isolated from Areca spathe by sulphuric acid hydrolysis. The morphological studies proved that the extracted fibres are of almost uniform diameter in the range $55-70 \mathrm{~nm}$. The mechanical processes help in the individualization of the fibres from agglomerated state. The composition analyses of raw fibres revealed that Areca spathe contain $64.5 \%$ holocellulose and $39 \% \alpha$ - cellulose. FTIR analysis results showed that non-cellulosic components remarkably decreased after consecutive stages of chemical treatment. The XRD results indicated that the crystallinity of fibres increased during sulphuric acid hydrolysis. Finally, based on the analysis results, it is clear that nanocellulose fibre with high crystallinity and uniform diameter can be isolated from Areca spathe by sulphuric acid hydrolysis.

\section{REFERENCES}

[1] Maheswari, C. Uma, K. Obi Reddy, E. Muzenda, B. R. Guduri, and A. Varada Rajulu. "Extraction and characterization of cellulose microfibrils from agricultural residueCocosnucifera L."Biomass and bioenergy 46, 2012, pp. 555-563.

[2] Wise, Louis Elsberg, Maxine Murphy, and A. A. D ADIECO."A chlorite holocellulose, its fractionation and bearing on summative wood analysis and studies on the hemicelluloses." 1946, pp. 35-43.

[3] Rowland, A. P., and J. D. Roberts. "Lignin and cellulose fractionation in decomposition studies using acid-detergent fibre methods." Communications in Soil Science and Plant Analysis 25(4), 1994, pp. 269-277.

[4] Nazir, Muhammad Shahid, Bambang Air Wahjoedi, Abdul Wahid Yussof, and Mohd Azmuddin Abdullah. "Eco-friendly extraction and characterization of cellulose from oil palm empty fruit bunches". BioResources, 8(2), 2013, pp. 2161-2172

[5] Sun, X. F., Feng Xu, R. C. Sun, P. Fowler, and M. S. Baird. "Characteristics of degraded cellulose obtained from steam-exploded wheat straw." Carbohydrate research 340(1), 2005, pp. 97-106.

[6] Hessien, M. M., M. M. Rashad, R. R. Zaky, E. A. Abdel-Aal, and K. A. El-Barawy. "Controlling the synthesis conditions for silica nanosphere from semi-burned rice straw." Materials science and engineering: B 162(1), 2009, pp. 14-21.

[7] Biagiotti, J., D. Puglia, and Jose M. Kenny. "A review on natural fibre-based compositespart I: structure, processing and properties of vegetable fibres." Journal of Natural Fibers 1(2), 2004, pp. 37-68. 
[8] Chandra Julie, Neena George, and Sunil K. Narayanankutty. "Isolation and characterization of cellulose nanofibrils from arecanut husk fibre." Carbohydrate polymers 142, 2016, pp. 158-166.

[9] Xiao, B., Sun, X, and Sun, R. "Chemical, structural, and thermal characterizations of alkalisoluble lignins and hemicelluloses, and cellulose from maize stems, rye straw, and rice straw". Polymer degradation and stability, 74(2), 2001, pp. 307-319

[10] de Rodriguez, Nancy Lis Garcia, Wim Thielemans, and Alain Dufresne. "Sisal Cellulose Whiskers Reinforced Polyvinyl Acetate Nanocomposites." Cellulose 13(3), 2006, pp. 261270.

[11] Abraham, E., B. Deepa, L. A. Pothan, M. Jacob, S. Thomas, U. Cvelbar, and R. Anandjiwala. "Extraction of Nanocellulose Fibrils from Lignocellulosic Fibres: A Novel Approach." Carbohydrate Polymers 86(4), 2011, pp. 1468-1475.

[12] Avolio, R., I. Bonadies, D. Capitani, M. E. Errico, G. Gentile, and M. Avella. "A multi technique approach to assess the effect of ball milling on cellulose." Carbohydrate polymers 87(1), 2012, pp. 265-273.

[13] Jonoobi, Mehdi, Abolghasem Khazaeian, Paridah Md Tahir, Syeed Saiful Azry, and Kristiina Oksman. "Characteristics of cellulose nanofibers isolated from rubberwood and empty fruit bunches of oil palm using chemo-mechanical process." Cellulose 18(4), 2011, pp. 1085-1095.

[14] Cherian, Bibin Mathew, Laly A. Pothan, Tham Nguyen-Chung, Günter Mennig, M. Kottaisamy, and Sabu Thomas. "A novel method for the synthesis of cellulose nanofibril whiskers from banana fibers and characterization."Journal of agricultural and food chemistry 56(14), 2008, pp. 5617-5627.

[15] Fatah, Ireana, H. P. S. A. Khalil, Md Hossain, Astimar Aziz, Yalda Davoudpour, Rudi Dungani, and Amir Bhat. "Exploration of a chemo-mechanical technique for the isolation of nanofibrillated cellulosic fiber from oil palm empty fruit bunch as a reinforcing agent in composites materials." Polymers 6(10), 2014, pp. 2611-2624.

[16] Nishiyama, Yoshiharu. "Structure and properties of the cellulose microfibril."Journal of wood science 55(4), 2009, pp. 241-249.

[17] Chirayil, Cintil Jose, Jithin Joy, Lovely Mathew, Miran Mozetic, Joachim Koetz, and Sabu Thomas. "Isolation and characterization of cellulose nanofibrils from Helicteresisora plant."Industrial Crops and Products 59, 2014, pp. 27-34.

[18] de Souza Lima, M. Miriam, and Redouane Borsali. "Rodlike cellulose microcrystals: structure, properties, and applications." Macromolecular rapid communications 25(7), 2004, pp. 771-787.

[19] Tang, Yanjun, Shujie Yang, Nan Zhang, and Junhua Zhang. "Preparation and characterization of nanocrystalline cellulose via low-intensity ultrasonic-assisted sulfuric acid hydrolysis." Cellulose 21(1), 2014, pp. 335-346

[20] Araki, Jun, Masahisa Wada, Shigenori Kuga, and Takeshi Okano. "Flow properties of microcrystalline cellulose suspension prepared by acid treatment of native cellulose." Colloids and Surfaces A: Physicochemical and Engineering Aspects 142(1), 1998, pp. 7582.

[21] Lu, Ping, and You-Lo Hsieh. "Preparation and properties of cellulose nanocrystals: rods, spheres, and network." Carbohydrate polymers 82(2), 2010, pp. 329-336.

[22] M. Mounika and Dr. K. Ravindra. Characterization of Banana Fiber/Pistacia Vera Shell Cellulose Reinforced Composites. International Journal of Mechanical Engineering and Technology, 8(2), 2017, pp. 123-130 
[23] B. Goutham, CH. Karunakar, A. Prashanth and T. Vamshi, Development and Evaluation of Some of the Mechanical Properties of Kenaf/Polyester Cellulose Composites, International Journal of Mechanical Engineering and Technology (IJMET), Volume 5, Issue 9, September (2014), pp. 259-265

[24] Abbas S. Al-Ameeri, Effect of Elevated Temperature on Some Properties of Technical Gypsum Reinforced by Cellulose Fiber, International Journal of Civil Engineering and Technology (IJCIET), Volume 5, Issue 4, April (2014), pp. 10-27

[25] Kiran Singh, T. Jai Mangal Sinha and Shalini Srivastava, Fabrication of SilaneNanocrystalline Cellulose: Bio-Nanocomposites for the Abatement of Arsenic, International Journal of Advanced Research in Engineering and Technology (IJARET), Volume 5, Issue 9, September (2014), pp. 70-79 